\title{
CorShrink : Empirical Bayes shrinkage estimation of correlations, with applications
}

\author{
Kushal K. Dey ${ }^{1}$, Matthew Stephens ${ }^{1,2}$
}

1 Department of Statistics, University of Chicago

2 Department of Human Genetics, University of Chicago

* Corresponding Email : kkdey@uchicago.edu

\begin{abstract}
Estimation of correlation matrices and correlations among variables is a ubiquitous problem in statistics. In many cases - especially when the number of observations is small relative to the number of variables - some kind of shrinkage or regularization is necessary to improve estimation accuracy. Here, we propose an Empirical Bayes shrinkage approach, CorShrink, which adaptively learns how much to shrink correlations by combining information across all pairs of variables. One key feature of CorShrink, which distinguishes it from most existing methods, is its flexibility in dealing with missing data. Indeed, CorShrink explicitly accounts for varying amounts of missingness among pairs of variables. Numerical studies suggest CorShrink is competitive with other popular correlation shrinkage methods, even when there is no missing data. We illustrate CorShrink on gene expression data from GTEx project, which suffers from extensive missing observations, and where existing methods struggle. We also illustrate its flexibility by applying it to estimate cosine similarities between word vectors from word2vec models, thereby generating more accurate word similarity rankings.
\end{abstract}

Keywords: correlation shrinkage, Empirical Bayes, missing data, genomics, word2vec

\section{Introduction}

Estimating the correlation matrix of a set of variables is a fundamental problem in statistics. The simplest estimator, the sample correlation matrix, can perform poorly when the number of variables $(p)$ is large compared with the number of samples $(n)$. This problem has motivated many alternative estimators, most of them based on shrinkage or regularization methods. These include using a convex combination of the sample correlation matrix with one or more target correlation matrices Lancewicki and Aladjem, 2014, Ledoit and Wolf, 2003, 2004, Schäfer and Strimmer, 2005, Touloumis, 2015]; thresholding-based approaches using either hard-thresholding Bickel and Levina, 2008 or soft-thresholding Rothman and others, 2009; and L1-penalized shrinkage on correlation or inverse correlation matrices [Bien and Tibshirani, 2011, Friedman and others, 2008]. These methods, though often a considerable improvement on the sample correlation matrix, have their own limitations. In particular, almost all of these approaches assume that there are no missing observations. Here we present CorShrink, a fast and simple shrinkage-based approach to estimating correlations that can deal 
with missing observations, and, in many settings, is competitive in accuracy with existing methods.

In brief, CorShrink is a model-based extension of the approaches of Bickel and Levina 2008 and Rothman and others 2009. Those approaches perform shrinkage using simple thresholding rules applied independently to each element of the correlation matrix. Our approach also treats each element of the matrix as independent, but uses an Empirical Bayes (EB) shrinkage approach that combines information across all elements to learn how much to shrink. Within this EB approach, the amount of shrinkage of each matrix element depends on the curvature of its likelihood, and so it naturally adapts to differing amounts of data available on each element. Our methods exploit semi-parametric EB methods Stephens, 2016] that are both computationally fast and stable, and avoid strong assumptions on the distributions of pairwise correlations.

We illustrate CorShrink on a dataset with extensive missingness: gene expression data across 53 human tissues (and cell-lines) collected by Genotype Tissue Expression (GTEx) project Lonsdale and others, 2013. The data matrix for each gene consists of expression measurements on many post-mortem donors across many tissues. However, for most donors, data are available on only a subset of tissues, and many elements of this data matrix are missing. Some tissues are particularly rarely sampled, and so their sample correlations are particularly noisy. Most existing correlation matrix estimation procedures cannot be easily applied to these data. In contrast, as we illustrate here, CorShrink is straightforward to apply. Further, the resulting shrinkage estimates are more biologically plausible, and visually considerably less cluttered, than the sample correlation matrix estimate. We also compare results with an alternative approach based on first imputing the missing values in the data matrix, and conclude that for data like these with high levels of missingness this imputation strategy is unappealing.

In addition to these results with missing data, we also use simple simulations to show that even when no data are missing, CorShrink is often competitive in accuracy with other methods, especially when the true correlation matrix is sparse. Furthermore, CorShrink is easy to apply to other correlation-like quantities, and we illustrate this by applying it to cosine similarities of vectors from word2vec models Mikolov and others, 2013 that measure word-word similarities.

\section{Methods}

Let $\left(X_{n p}\right)_{N \times P}$ denote a data matrix with $N$ samples and $P$ variables, where some values may be missing (recorded as NA). For each pair of variables $i, j \in\{1,2, \cdots, P\}$ let $R_{i j}$ denote their (unknown) true correlation, and $\hat{R}_{i j}$ denote the sample correlation computed using only the samples $n$ that have observed values for both the variables $i$ and $j$ (e.g. using the option use="pairwise.complete.obs", method = "pearson" in the R function cor). Further, let $Z_{i j}$ and $\hat{Z}_{i j}$ denote the corresponding Fisher Z-transforms Fisher, 1915:

$$
\begin{aligned}
& Z_{i j}=Z\left(R_{i j}\right)=\frac{1}{2} \log \left(\frac{1+R_{i j}}{1-R_{i j}}\right) \\
& \hat{Z}_{i j}=Z\left(\hat{R}_{i j}\right) .
\end{aligned}
$$

Our goal is to estimate the matrix $Z=\left(Z_{i j}\right)$ and hence $R=\left(R_{i j}\right)$ from the data $\hat{Z}=\left(\hat{Z}_{i j}\right)$.

Our starting point is a result from Fisher 1921, who showed that, under a bivariate normality assumption on each pair of variables, the observations $\hat{Z}_{i j}$ are approximately normal:

$$
\hat{Z}_{i j} \mid Z_{i j} \sim N\left(Z_{i j}, s_{i j}\right) \quad \forall i<j .
$$


Here the standard deviation $s_{i j}$ is given by

$$
s_{i j}=\sqrt{\frac{1}{\left(n_{i j}-1\right)}+\frac{2}{\left(n_{i j}-1\right)^{2}}},
$$

where $n_{i j}>3$ is the number of matched samples, for which both variables $i$ and $j$ are observed:

$$
n_{i j}:=\#\left\{n: X_{n i} \neq \mathrm{NA}, \quad X_{n j} \neq \mathrm{NA}\right\} .
$$

We take an Empirical Bayes approach, combining the likelihood (3) with an assumption that the $Z_{i j}$ are drawn from some underlying distribution $g$ :

$$
Z_{i j} \sim g \in \mathcal{G}
$$

where $\mathcal{G}$ denotes some pre-specified family of distributions. There are many possible choices for $\mathcal{G}$, and here we make use of the flexible nonparametric methods implemented in the $\mathrm{R}$ package ashr Stephens, 2016. These include:

1. $\mathcal{G}=\mathcal{N} \mathcal{P}$, the set of all real-valued distributions. This is the fully non-parametric approach from Koenker and Mizera 2014;

2. $\mathcal{G}=\mathcal{U}$ the set of all unimodal distributions;

3. $\mathcal{G}=\mathcal{S U}$ the set of all symmetric unimodal distributions;

4. $\mathcal{G}=\mathcal{U}_{+}$(respectively $\mathcal{G}=\mathcal{U}_{-}$) the set of unimodal distributions that are constrained to be positive (respectively negative).

5. $\mathcal{G}=\mathcal{S N}$ the set of all scale-mixtures of normals.

The rationale for the more restrictive unimodal choices $\left(\mathcal{U}, \mathcal{S U}, \mathcal{U}_{+}, \mathcal{S N}\right)$ is that they ensure shrinkage towards a common point, and also regularize the estimate of $g$ compared with a fully non-parametric approach. Stephens 2016 focused on the case where the mode of $g$ was at 0 , but the ashr package also implements methods to estimate the location of the mode, which may also be useful in this context. Results in this paper were generated using $\mathcal{G}=\mathcal{U}$ with mode at 0 unless otherwise stated.

We now make two simplifying assumptions: that the $Z_{i j}$ are independent from (6) and $\hat{Z}_{i j} \mid Z_{i j}$ are independent from (3). Neither of these assumptions is true, but composite likelihood theory [Varin and others, 2011] suggests that point estimates of both $g$ and $Z$ should be somewhat robust to dependence, and our numeric experiments later show that point estimates from our method can outperform methods that do model the dependence. These simplifying assumptions can be seen as a model-based analogue of previous methods that apply simple thresholding rules independently to each element of the correlation matrix [Bickel and Levina, 2008, Rothman and others, 2009].

We fit the above model to obtain the posterior mean of $Z_{i j}, Z_{i j}^{\star}$, given $\dot{R}_{i j}$ :

$$
Z_{i j}^{\star}:=E\left[Z_{i j} \mid \hat{R}_{i j}\right]
$$

$Z_{i j}^{\star}$ are adaptively shrunk estimates of $\hat{Z}_{i j}$ that account for $n_{i j}$, the number of matched samples between variables $i$ and $j$. The smaller the $n_{i j}$, the larger would be $s_{i j}$ in Equation 4 and higher would be the level of shrinkage on $\hat{Z}_{i j}$.

Next, we reverse transform $Z_{i j}^{\star}$ to correlation estimate $R_{i j}^{\star}$ :

$$
R_{i j}^{\star}:=\frac{\exp \left(2 Z_{i j}^{\star}\right)-1}{\exp \left(2 Z_{i j}^{\star}\right)+1} .
$$


Just as with simple threholding methods the matrix $R^{\star}=\left(\left(R_{i j}^{\star}\right)\right)_{P \times P}$ may not be positive definite, so we select the nearest positive definite matrix $R^{\star \star}$ to $R^{\star}$, using the method from Higham 2002.

If the variables are not pairwise normally distributed, then the representation of $s_{i j}$ as in Equation (4) does not hold. One approach in this context is to use

transformations of the data that are more robust to the non-normality of the data, for example - Box-cox, ranks, rank-based inverse normal (RIN) transformations Bishara and Hittner, 2015, 2017]. Another approach would be to estimate $s_{i j}$ using Bootstrap methods Diaconis and Efron, 1983, Efron, 1981]. The flexibility to use re-sampling methods as above, extends the scope of the CorShrink method beyond correlations to any correlation-like quantities -partial correlations, rank correlations, cosine similarities between word vectors in a word2vec model etc.

\section{Results}

\subsection{Applications - Genetics}

We first illustrate CorShrink on a data set with many missing observations. The Genotype Tissue Expression (GTEx) Project Lonsdale and others, 2013 collected gene expression data from hundreds of post-mortem donors across many different tissues.

The version of the data we consider here (v6) contains $\sim 540$ donors across 51 different tissues and 2 cell lines. If every donor contributed every tissue then the expression data at each gene would be a 540 by 53 matrix, each entry of which is a real number (a measure of expression known as log-counts-per-million; Law and others 2014]).

Different donors, however, contributed different tissues leading to a large number of missing observations $(>70 \%)$ in this matrix.

Figure 1 illustrates CorShrink using data from a single gene, PLIN1. Panel (a) shows the pairwise sample correlation between tissues, which are input to CorShrink. One notable feature of these sample correlations is strong positive correlation among brain-derived tissues. Another feature is that many of the sample correlations are quite strongly negative. Biologically, strong negative correlation among tissues are expected to be rare, so these observations may be driven primarily by sampling variation. This view is further supported by noting that the most negative values tend to occur in tissue pairs that have fewer matching samples (e.g. nerve-tibial and cervix-endocervix have correlation -0.89 with only 3 matching samples). Panel (b) shows the estimated $\hat{g}$, with $\mathcal{G}=\mathcal{U}$ and the mode fixed to 0 . The estimate is skewed towards small positive values, consistent with the biologically plausible scenario that most pairs of tissues are mildly positively correlated. Panel (c) shows the resulting CorShrink shrinkage estimates of the correlation. The sample correlations are all shrunk towards the mode of $g$, but the amount of shrinkage for each tissue pair depends on matched sample sizes: pairs with few matched samples undergo strong shrinkage while those with more matched samples remain largely unperturbed (Panel $(\mathrm{d})$ ). The negative sample correlations are mostly removed in the shrinkage estimates - due to both the concentration of $\hat{g}$ on positive values, and the stronger shrinkage of tissue pairs with small matched sample size.

Given the biological expectation that negative correlations among tissues are unlikely, we might prefer to constrain the correlations to be positive here by using $\mathcal{G}=\mathcal{U}_{+}$. The results for this, in addition to several other choices of $\mathcal{G}$ are shown in Supplementary Figure S1. The results in this case are largely consistent across these choices of $\mathcal{G}$, although only $\mathcal{G}=\mathcal{U}_{+}$completely removes negative estimates.

These illustrative results were "gene-based" - that is, they were based on data at the PLIN1 gene alone, and involved shrinking correlations for all tissue-pairs using the same prior distribution $\hat{g}$. However, for some tissue pairs (e.g. biologically-related tissues, like 
(a)

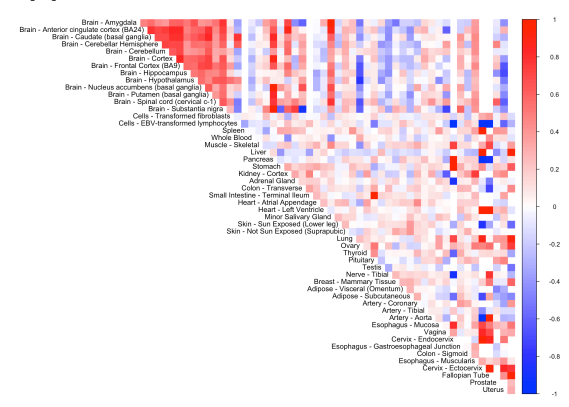

(c)

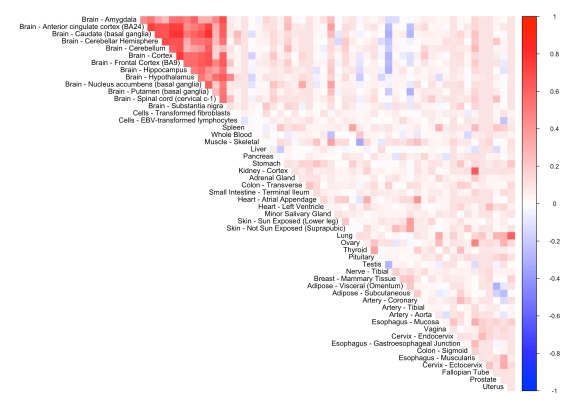

(b)
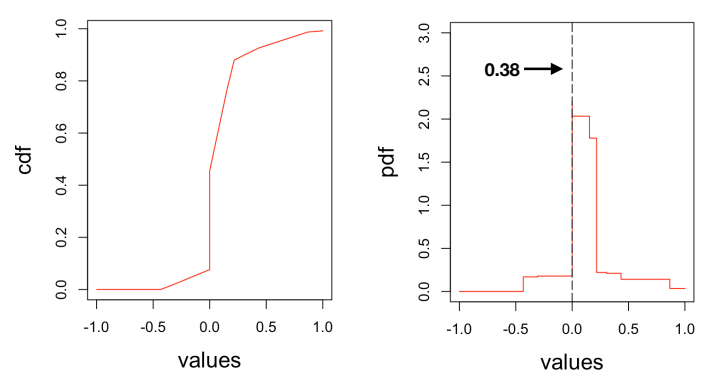

(d)

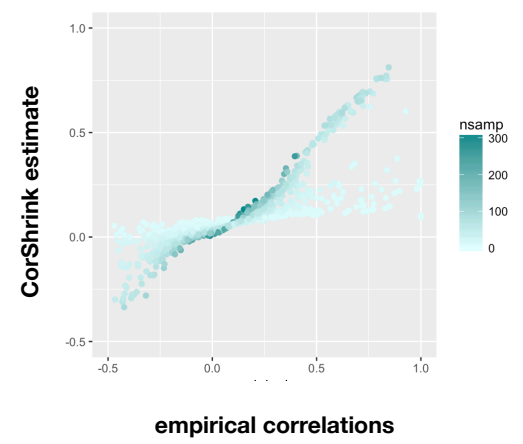

Figure 1. Illustration of CorShrink in action. (a) The image plot of the pairwise correlation matrix between tissue pairs for the log CPM expression data Law and others, 2014 of the PLIN1 gene. (b) The probability and cumulative density function plots for the EB shrinkage prior in CorShrink generated using $\mathcal{G}=\mathcal{U} .0 .38$ is the empirically determined prior probability mass of observing 0 correlation. (c) The image plot of the estimated correlation matrix using CorShrink. The representation is visually more parsimonious and arguably more interpretable than (a). (d) Scatter plot of the pairwise sample correlation values against the CorShrink fitted estimates for all tissue pairs. Each point is colored by the number of postmortem donors contributing the corresponding tissue pair. Expectedly, the pairs with low numbers of matching samples (light colored points) undergo high shrinkage while those with larger number of matching samples remain largely unperturbed. 
the two heart tissues) strong correlations are more plausible than for others. Here, since we have data on many genes for each tissue pair, we can instead separately estimate a prior distribution $\hat{g}$ for each tissue pair, by combining data across genes. That is, for each tissue pair, we feed into Equation 3 the vector of pairwise correlation in expression for this tissue pair across all genes, together with their corresponding standard errors. Supplementary Figure S2 shows results from this "tissue-pair-based shrinkage" with the "gene-based shrinkage" in Figure 1. The two results are reassuringly similar, especially when compared with the original sample correlations.

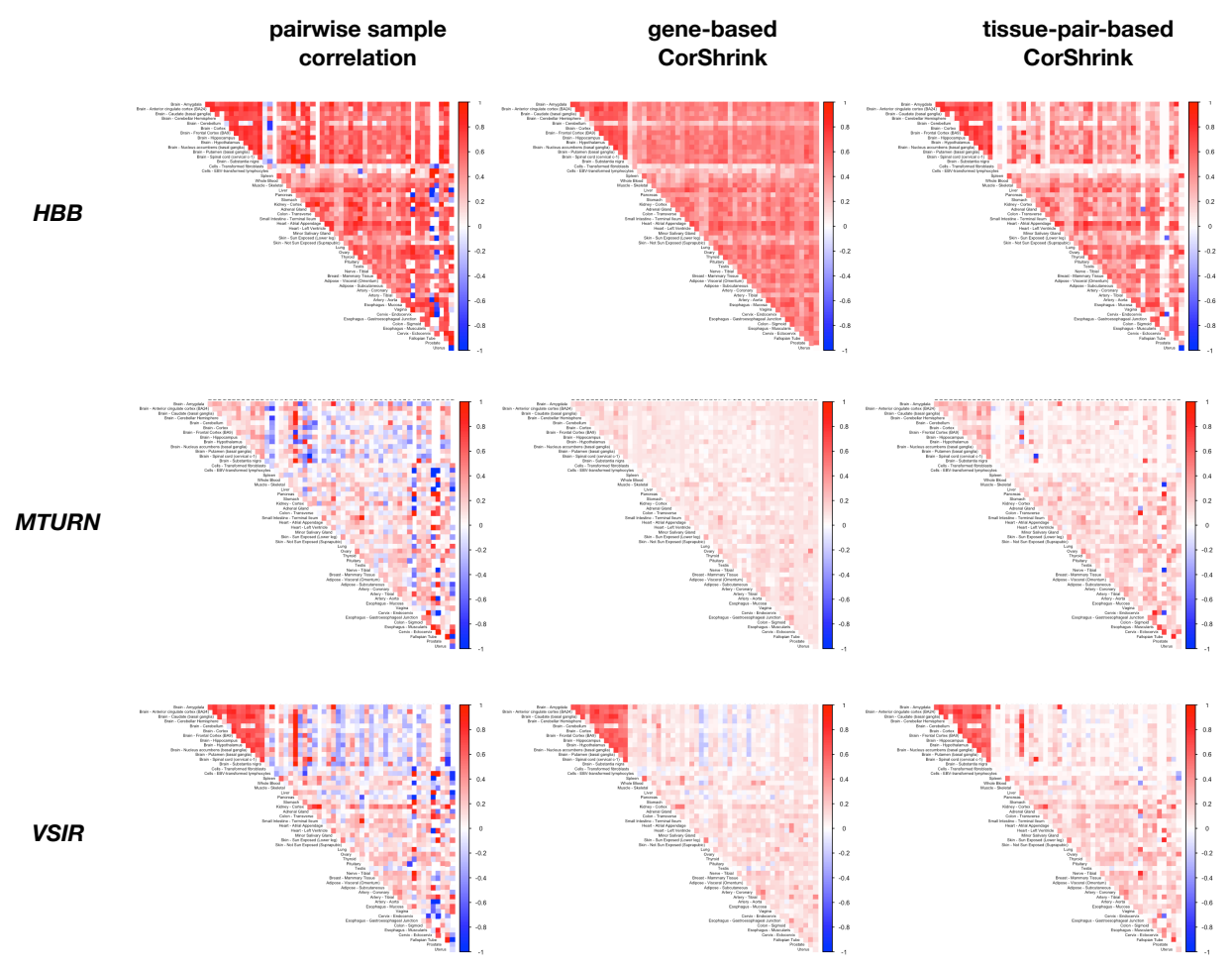

Figure 2. Results from CorShrink applied to three different genes $(H B B, M T U R N, V S I R)$ showing qualitatively different patterns of correlation. The gene-based results illustrate how CorShrink adapts itself to different amounts of correlation in different genes (e.g. compare the modest shrinkage for $H B B$ with the stronger shrinkage for $M T U R N)$. See text for further discussion.

To further illustrate the flexibility of CorShrink, Figure 2 shows results for three different genes that show different qualitative patterns of correlation. One gene (VSIR) shows correlation patterns similar to those for PLIN1 above, with strong correlations mostly among brain tissues; this type of pattern occurs in many genes in this data set (not shown). In contrast the $H B B$ gene (which encodes beta-globin, a sub-unit of hemoglobin) shows high correlations across many tissue pairs - both brain, and non-brain. We noticed similar patterns in other hemoglobin-related genes, $H B A 1$ and HBA2 (not shown). Finally, the gene $M T U R N$ shows little correlation in expression across most pairs of tissues. These results particularly highlight the adaptiveness of the gene-based analysis to gene-specific patterns. Effectively CorShrink learns that the sample correlations in MTURN are mostly consistent with weak correlation, and so shrinks these sample correlations strongly toward 0. By comparison the shrinkage in $H B B$ is considerably less pronounced.

One important feature of CorShrink is its ability to deal with a high rate of missing 
(a)

correlation on softImpute -imputed data

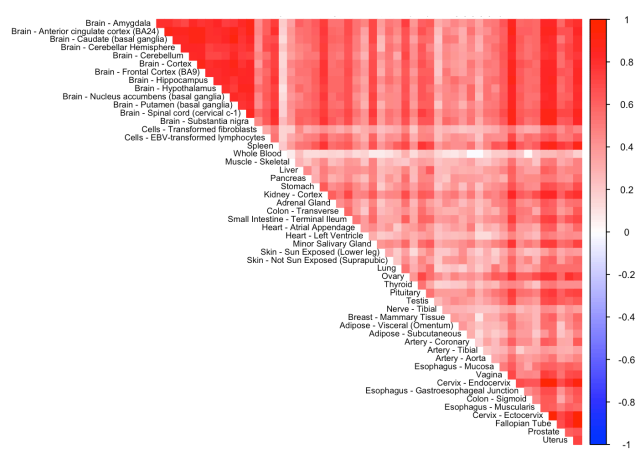

(b)

correlation on FLASH-imputed data

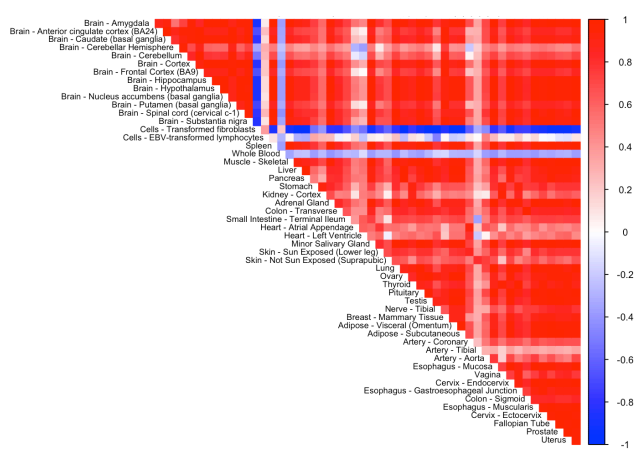

Figure 3. Illustration of impact of imputation on correlation matrix estimates. The panels show sample correlation matrices for the same data (PLIN1) as in Figure 1, after imputation using (a) softImpute [Mazumder and others, 2010] and (b) FLASH Wang and Stephens 2018. In both cases the results appear inconsistent with the observed data, unlike the results from CorShrink (Figure 1,a,c) 
data $(>70 \%$ in these examples). One general and widely-used approach to deal with missing data is to impute them, for example by using low-rank-based methods such as softImpute Hastie and Mazumder, 2015, Mazumder and others, 2010 or FLASH Wang and Stephens, 2018. However, we have found that in this context, with its high rate of missing data, imputation can grossly distort correlation estimates. For example, Figure 3 illustrates the dramatic effects of imputation for PLIN1. The imputed data from both softImpute and FLASH show a strong upward bias in their correlation for most tissue pairs, which appears inconsistent with the observed pairwise correlations.

\subsection{Simulation studies}

Although CorShrink is particularly well-suited to settings with missing data, it is also a very competent correlation shrinkage method for complete data. Here we demonstrate this using simulations to compare CorShrink with other correlation shrinkage methods: soft thresholding estimator PDSCE [Rothman, 2012] and corpcor, which performs shrinkage towards the identity matrix [Schäfer and Strimmer, 2004, 2005]. We also compare with GLASSO Friedman and others, 2008, Meinshausen and Buhlmann, 2006, Witten and others, 2011, which is based on inducing sparsity in the precision matrix.

We simulated multivariate normal data with 0 mean and three types of correlation structure: a hub correlation matrix (sparse correlation, sparse precision), a Toeplitz correlation matrix (sparse correlation, non-sparse precision) and a banded precision matrix (non-sparse correlation, sparse precision). In each case we simulated $p=100$ features for sample size $n=30,50,100,1000$. We ran CorShrink with $\mathcal{G}=\mathcal{S N}$; we ran GLASSO at varying levels of the tuning parameter $\lambda=0.01,0.1,0.5$ and 1 .

Figure 4 compares the accuracy of each method in estimating the correlation matrix, as measured by Correlation Matrix Distance (CMD) Herdin and others, 2005 between the true and estimated matrix. For both the hub and Toeplitz scenarios CorShrink was consistently the most accurate, and for the sparse banded precision matrix case it performed similarly to the best-performing of the GLASSO approaches. Similar results hold if accuracy is measured using Frobenius distance instead of CMD (Supplementary Figure S3.

Figure 5 compares, for each method, the largest eigenvalues of the estimated correlation matrices with those of the population correlation matrix. Again, for hub and Toeplitz scenarios, CorShrink estimates consistently follow the population eigenvalues more closely than other methods.

In some applications interest may be focused on estimating the inverse of the correlation matrix, or the partial correlation matrix, rather than the correlation matrix itself. As currently implemented CorShrink is not well suited to such applications: in particular the correlation matrix estimate from CorShrink is typically close to singular when $n<<p$, so its inverse is unstable, and does not generally provide a good estimate for the inverse-correlation matrix (in Frobenius norm, say). In contrast GLASSO is specifically designed for estimating (sparse) inverse correlation matrices, and indeed for this task GLASSO outperforms the other methods considered here on our examples (Supplementary Figure S4, which all involve sparse precision matrices.

\subsection{Applications - Natural Language Processing}

CorShrink can also be applied to perform shrinkage estimation of other correlation-like quantities. Here we illustrate this in an application from Natural Language Processing: estimating cosine similarities between vector representations of words, as obtained from a word2vec Mikolov and others, 2013 or GLOVE [Pennington and others model for example. The aim here is to generate more robust estimates of the cosine similarities 


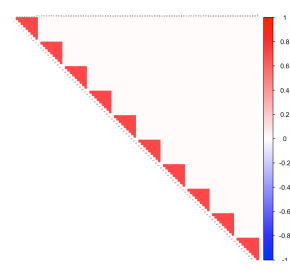

Hub correlation

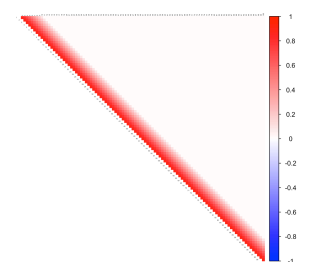

Toeplitz correlation

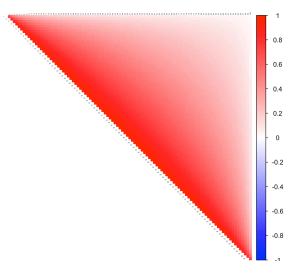

Banded precision
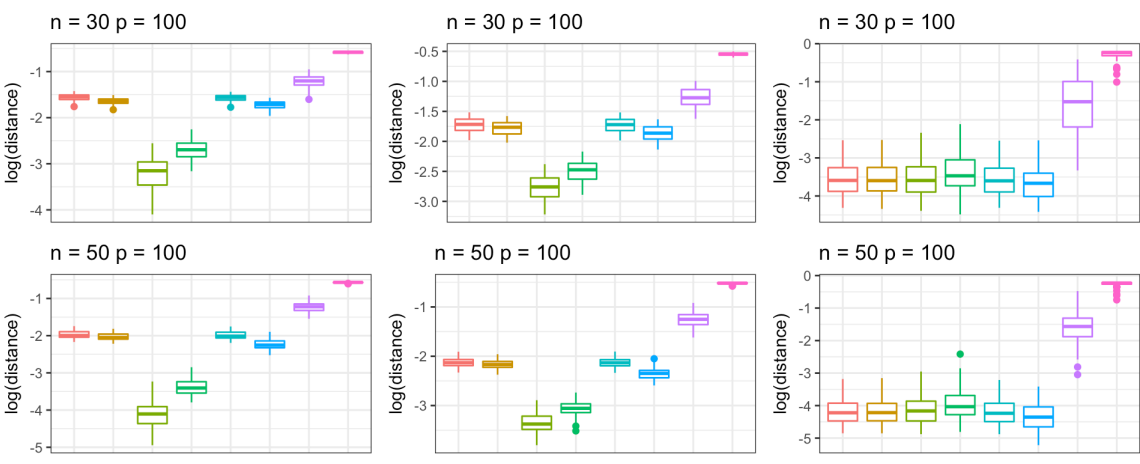

method

它 empirical

宁 corpcor

由 corshrink

$n=100 p=100$

$n=100 p=100$

$n=100 p=100$
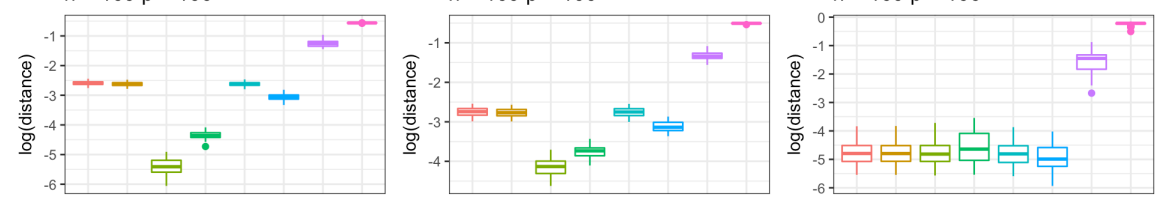

审 glasso:1e-02

官 glasso:0.1

审 glasso:0.5

官 glasso:1
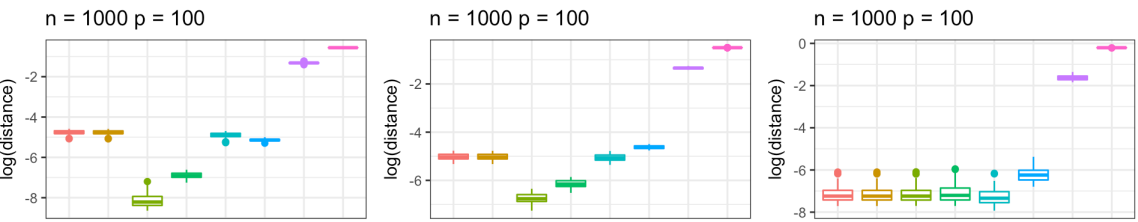

Figure 4. Box plot of the Correlation Matrix Distance (CMD) Herdin and others, 2005 between population correlation matrix and the estimated matrix from different methods - corpcor [Schäfer and Strimmer, 2004, 2005], CorShrink, PDSCE [Rothman, 2012], GLASSO [Friedman and others, 2008 and the empirical pairwise correlation matrix. Three types of population correlation structure were considered - hub structure, Toeplitz structure and the banded precision matrix structure. Image plots for these population correlation matrices are shown in the topmost panel. GLASSO was fitted using a broad range of tuning parameters $(\lambda=0.01,0.1,0.5$ and 1$)$, and their relative performance was also compared. CorShrink outperforms the other methods for the sparse structured population correlation model examples (hub and Toeplitz), closely followed by $P D S C E$. 


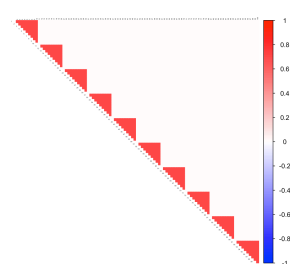

Hub correlation
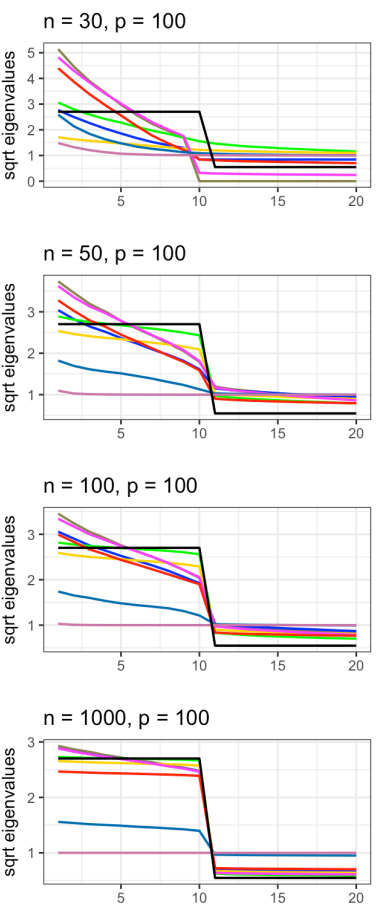

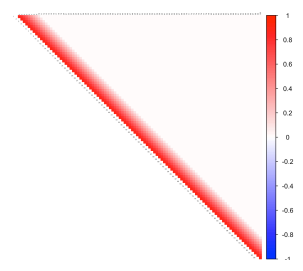

Toeplitz correlation

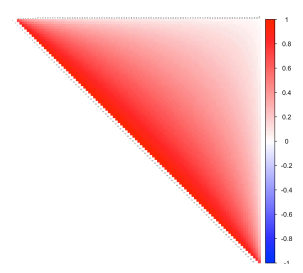

Banded precision
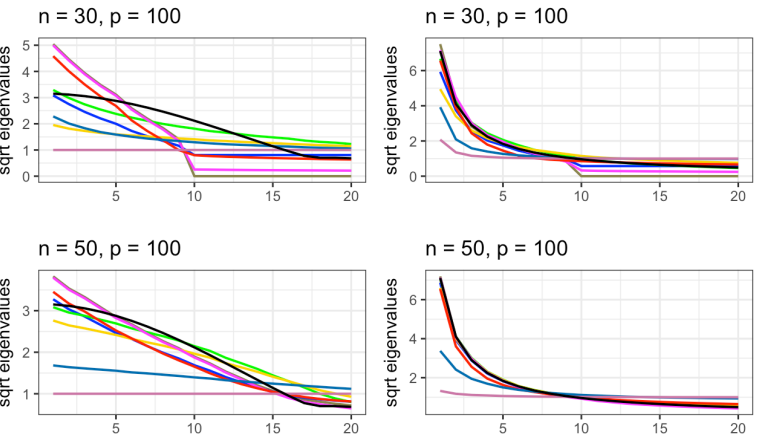

- empirical - corpcor - corshrink - pdsce - glasso:1e-02
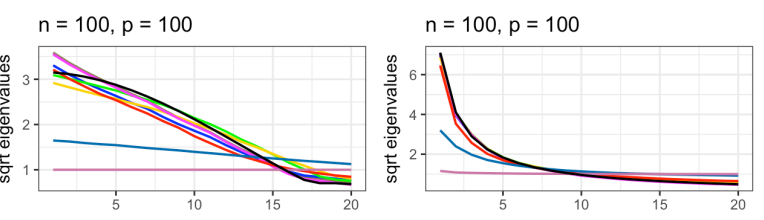

— glasso:0.1

— glasso:0.5

- glasso: 1

- true

Figure 5. Plots of sorted square-root eigenvalue trends of the population correlation matrix against those of the estimated correlation matrices using different methods - corpcor Schäfer and Strimmer, 2004, 2005, CorShrink, PDSCE Rothman, 2012, GLASSO [Friedman and others, 2008] and the empirical pairwise correlation matrix. Three types of population correlation structure were considered - hub structure, Toeplitz structure and the banded precision matrix structure. Image plots for these population correlation matrices are shown in the topmost panel. GLASSO was fitted using a broad range of tuning parameters $(\lambda=0.01,0.1,0.5$ and 1$)$, and their relative performance was also compared. The trend of sorted eigenvalues from CorShrink more closely follow the sorted population eigenvalues, in particular for the hub and the Toeplitz model examples. 
between words, less affected by sampling variation due to some words appearing relatively infrequently in a corpus.

We obtained text data from the monthly issues of Ebony magazine published in 1968. This year marked a major moment in American history with the assassination of Dr. Martin Luther King (MLK) and the subsequent effect on the civil rights movement, and these events are reflected in many of the articles. We fitted word2vec on the combined text data from these issues, obtained vector representations and computed (sample) cosine similarities between words based on the vector representations. We transformed these to Fisher Z-scores using (1).

Unlike correlations, there is no obvious way to compute standard errors of the Fisher Z-scores for these cosine similarities. Consequently we used re-sampling methods specifically 100 bootstrap samples Diaconis and Efron, 1983, Efron, 1981], with magazine issues being the sampling unit - to estimate standard errors. We used issues rather than articles as the sampling unit to preserve the feature that each issue typically contains a range of different article types (e.g. on sports, politics, lifestyle etc).
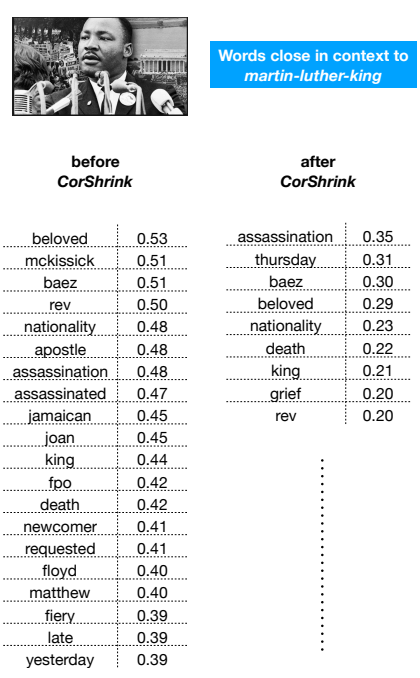
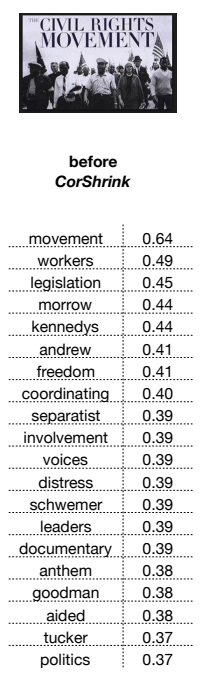
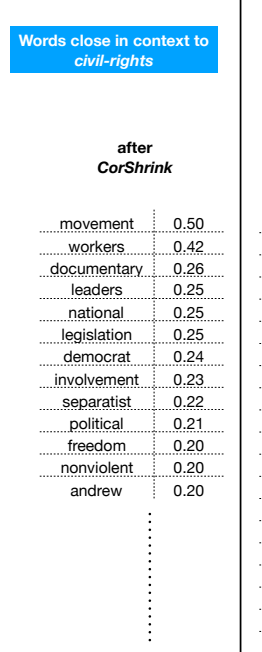

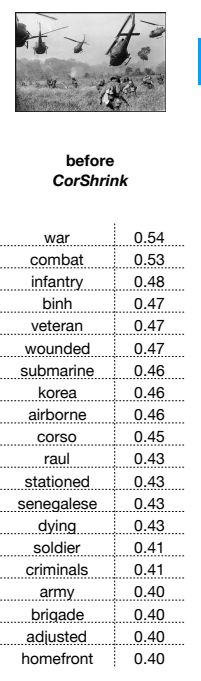

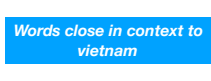

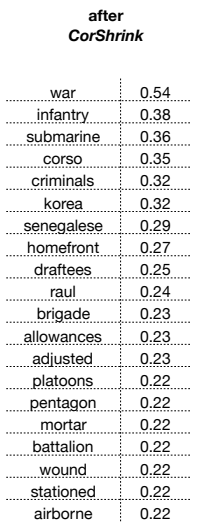

Figure 6. Results of CorShrink applied to estimates of cosine similarities between words. The tables show the top $\leq 20$ words estimated to be most similar to the word/phrase (s) martin-luther-king (left), civil-rights (middle) and vietnam (right), before and after applying CorShrink. Only words with estimated similarity score $>0.2$ are shown. The "before CorShrink" results were generated by running word2vec Mikolov and others, 2013] on the monthly issues of the Ebony magazine in the year of 1968. Words/phrases that occur infrequently in the context of the focal word/phrase are shrunk more strongly by CorShrink.

We focused on three words/phrases: martin luther king, civil rights and vietnam, treating each as a single "word" for our analysis. For each of these phrases we applied CorShrink to the corresponding Fisher $Z$-scores, with re-sampling standard errors computed as above. Figure 6 shows the top words contextually similar to the focal phrases based on cosine similarities before and after CorShrink shrinkage.

As expected, the cosine similarities after CorShrink are consistently smaller, due to shrinkage towards 0 . However, CorShrink also reorders the similarities because some words are shrunk more strongly than others. For example, the word apostle, which is strongly connected to the phrase martin-luther-king before CorShrink (ranked 6), has its cosine similarity shrunk strongly (from cosine similarity 0.48 to 0.13 ). This strong shrinkage occurs because the word occurred overall in 5 issues, and in only one of these 
issues was it very strongly associated with MLK (in the context of eulogizing him, immediately following his death in the May 1968 issue). In contrast, Thursday, which was the day of MLK's assassination, was shrunk much less strongly (from cosine similarity 0.38 to 0.31 ), because it occurs overall in 2 issues and consistently in connection with MLK in the May 1968 issue.

This shrinkage helps to highlight the words that are truly very strongly associated. For example, in connection with MLK the word assassination appears much higher up the list post shrinkage (moving from 7th to 1st). And although the word war is very strongly associated with vietnam both before and after shrinkage, its connection is clearer post-shrinkage because other words like combat and infantry are shrunk more strongly.

\section{Discussion}

We presented CorShrink, an application of the adaptive shrinkage framework from Stephens, 2016] to shrinkage estimation of correlation matrices and related quantities. Unlike most correlation matrix estimation approaches (corpcor, GLASSO), CorShrink can deal with missing values in the data matrix, and in this setting adjusts the degree of shrinkage based on the number of observations contributing to each matrix element (Figure 1). Even with no missing data, CorShrink can outperform the other methods when the true correlation matrix is sparse (see Figures 4.5. 5. In addition CorShrink has the flexibility to be applied to any set of correlation-like quantities, and not only correlation matrices. Here we illustrated this by shrinking cosine similarities between word pairs from text data (Figure 6).

The basic strategy underlying CorShrink is very simple, and involves shrinking each correlation term independently (after using all terms to learn how much to shrink). A similar simple strategy is also used in corpcor, and Bickel and Levina 2008 provide theoretical results for another (even simpler) strategy of this form. The simplicity of this strategy is very attractive, and is what allows CorShrink to easily deal with missing data for example. However, when the true correlation matrix is highly structured - for example, low rank plus diagonal - then methods that exploit this structure Fan and others, 2016 may be more accurate.

In terms of speed, CorShrink is comparable to corpcor, and considerably faster than glasso and PDSCE when one takes account of the need to tune these methods using cross-validation. As noted in the Results, the current implementation of CorShrink is not suited to estimating inverse correlations, and it may be interesting to consider whether modifications to the approach could rectify this. CorShrink is available as an $\mathrm{R}$ package on CRAN and is also under active development on Github https://github.com/kkdey/CorShrink; code implementing the analyses presented here are available at https://kkdey.github.io/CorShrink-pages/.

\section{References}

Bickel, Peter J and Levina, Elizaveta. (2008). Covariance regularization by thresholding. The Annals of Statistics, 2577-2604.

Bien, Jacob and Tibshirani, Robert J. (2011). Sparse estimation of a covariance matrix. Biometrika 98(4), 807-820.

BisharA, A.J. And Hittner, J.B. (2015). Reducing bias and error in the correlation coefficient due to nonnormality. Educational and psychological measurement $\mathbf{7 5 ( 5 ) ,}$ $785-804$. 
Bishara, A.J. And Hittner, J.B. (2017). Confidence intervals for correlations when data are not normal. Behavior research methods 49(1), 294-309.

Diaconis, P. And Efron, B. (1983). Computer-intensive methods in statistics. Scientific American 248(5), 116-131.

EFron, B. (1981). Nonparametric estimates of standard error: the jackknife, the bootstrap and other methods. Biometrika 68(3), 589-599.

FAN, J., LiaO, Y. AND LiU, H. (2016). Approaches to high-dimensional covariance and precision matrix estimation. Financial Signal Processing and Machine Learning, $100-134$.

Fisher, R.A. (1915). Frequency distribution of the values of the correlation coefficient in samples from an indefinitely large population. Biometrika 10(4), 507-521.

FisheR, R.A. (1921). On the probable error of a coefficient of correlation deduced from a small sample. Metron 1, 3-32.

Friedman, J., Hastie, T. and Tibshirani, R. (2008). Sparse inverse covariance estimation with the graphical lasso. Biostatistics 9(3), 432-441.

Hastie, T. and Mazumder, R. (2015). softimpute: Matrix completion via iterative soft-thresholded svd. $R$ package version, 1 ..

Herdin, M., Czink, N., Ozcelik, H. and Bonek, E. (2005). Correlation matrix distance, a meaningful measure for evaluation of non-stationary mimo channels. In Vehicular Technology Conference, 2005. VTC 2005-Spring. 2005 IEEE 61st 1, 136-140.

Higham, N.J. (2002). Computing the nearest correlation matrix-a problem from finance. IMA journal of Numerical Analysis 22(3), 329-343.

Koenker, R. And Mizera, I. (2014). Convex optimization, shape constraints, compound decisions, and empirical bayes rules. Journal of the American Statistical Association 109(506), 674-685.

Lancewicki, Tomer and Aladjem, Mayer. (2014). Multi-target shrinkage estimation for covariance matrices. IEEE Transactions on Signal Processing 62(24), 6380-6390.

LaW, C.W., Chen, Y., Shi, W. and Smyth, G.K. (2014). voom: Precision weights unlock linear model analysis tools for rna-seq read counts. Genome biology 15(2), R29.

Ledoit, Olivier And Wolf, Michael. (2003). Improved estimation of the covariance matrix of stock returns with an application to portfolio selection. Journal of empirical finance 10(5), 603-621.

Ledoit, Olivier And Wolf, Michael. (2004). A well-conditioned estimator for large-dimensional covariance matrices. Journal of multivariate analysis $\mathbf{8 8}(2)$, $365-411$.

Lonsdale, J., Thomas, J., Salvatore, M., Phillips, R., Lo, E., Shad, S., Hasz, R., Walters, G., Garcia, F., Young, N. and others. (2013). The genotype-tissue expression (gtex) project. Nature genetics 45(6), 580 . 
Mazumder, Rahul, Hastie, Trevor and Tibshirani, Robert. (2010). Spectral regularization algorithms for learning large incomplete matrices. Journal of machine learning research 11(Aug), 2287-2322.

Meinshausen, N. And Buhlmann, P. (2006). High-dimensional graphs and variable selection with the lasso. The annals of statistics, 1436-1462.

Mikolov, T., Sutskever, I., Chen, K., Corrado, G.S. and Dean, J. (2013). Distributed representations of words and phrases and their compositionality. In Advances in neural information processing systems, 3111-3119.

Pennington, J., Socher, R. And Manning, C.D. Glove: Global vectors for word representation.

Rothman, A J. (2012). Positive definite estimators of large covariance matrices. Biometrika 99(3), 733-740.

Rothman, Adam J, Levina, Elizaveta and Zhu, Ji. (2009). Generalized thresholding of large covariance matrices. Journal of the American Statistical Association 104(485), 177-186.

Schäfer, Juliane And Strimmer, Korbinian. (2004). An empirical bayes approach to inferring large-scale gene association networks. Bioinformatics 21(6), 754-764.

Schäfer, Juliane and Strimmer, Korbinian. (2005). A shrinkage approach to large-scale covariance matrix estimation and implications for functional genomics. Statistical applications in genetics and molecular biology 4(1).

Stephens, M. (2016). False discovery rates: a new deal. Biostatistics 18(2), 275-294.

Touloumis, Anestis. (2015). Nonparametric stein-type shrinkage covariance matrix estimators in high-dimensional settings. Computational Statistics $\&$ Data Analysis 83, 251-261.

Varin, C., Reid, N. And Firth, D. (2011). An overview of composite likelihood methods. Statistica Sinica 21, 5-42.

Wang, W. And Stephens, M. (2018). Empirical bayes matrix factorization. aRxiv, 1802.06931.

Witten, D.M., Friedman, J.H. and Simon, N. (2011). New insights and faster computations for the graphical lasso. Journal of Computational and Graphical Statistics 20(4), 892-900. 


\section{Supplementary Figures}

(a)

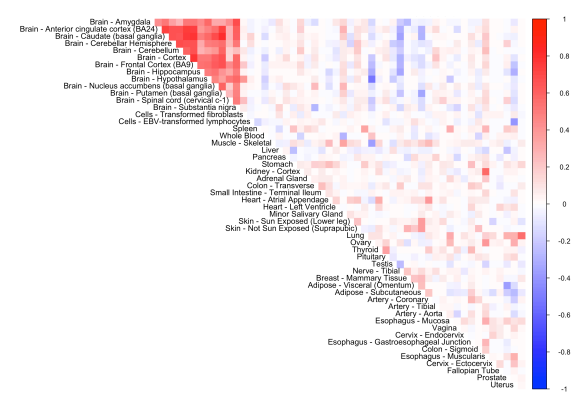

(b)

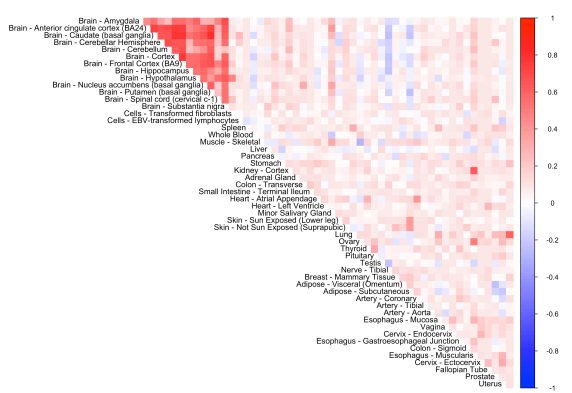

(c)

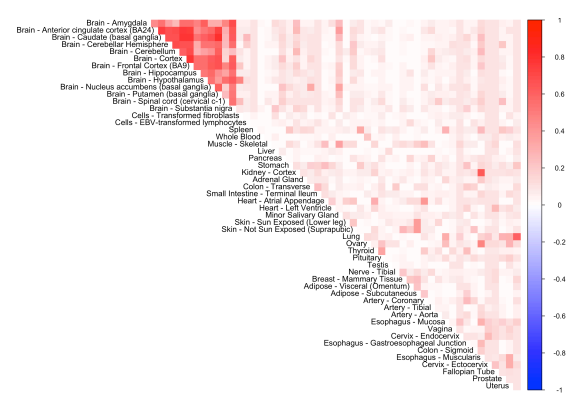

(d)

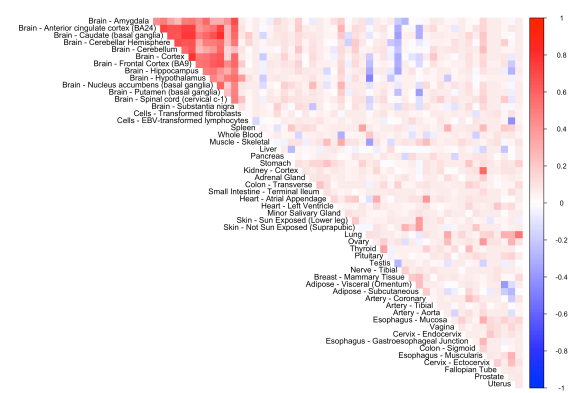

Figure S1. Image plots from applying CorShrink shrinkage on the donor by tissue expression data for PLIN1 gene using choices of $\mathcal{G}$ (in Equation 6) other than $\mathcal{G}=\mathcal{U}$ already reported in Fig 1 . (a) $\mathcal{G}=\mathcal{S U}_{(0)}$ where 0 is the mode of the distribution. (b) $\mathcal{G}=\mathcal{S U}_{(\hat{\mu})}$ where the mode $\hat{\mu}$ is not necessarily 0 and is estimated from the data. (c) $\mathcal{G}=\mathcal{U}_{+}$and $(\mathrm{d}) \mathcal{G}=\mathcal{N} \mathcal{P}$. See text for explanation of terminology and discussion. 

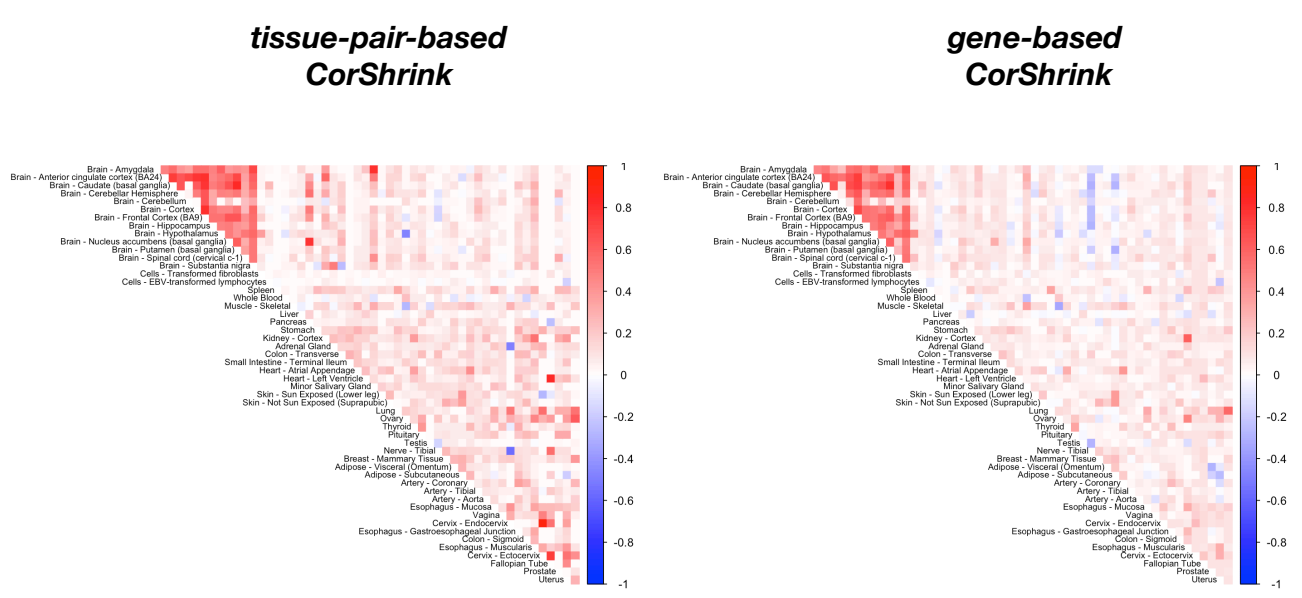

Figure S2. Image plots of the estimated correlation matrices after applying CorShrink on the subject by tissue expression matrix data for the PLIN1 gene for two different models - (a) "tissue-pair-based shrinkage" and (b) "gene-based shrinkage" models and with $\mathcal{G}=\mathcal{U}$. Both representations are re-assuringly similar. 


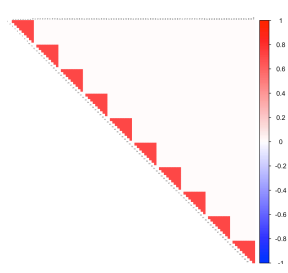

Hub correlation
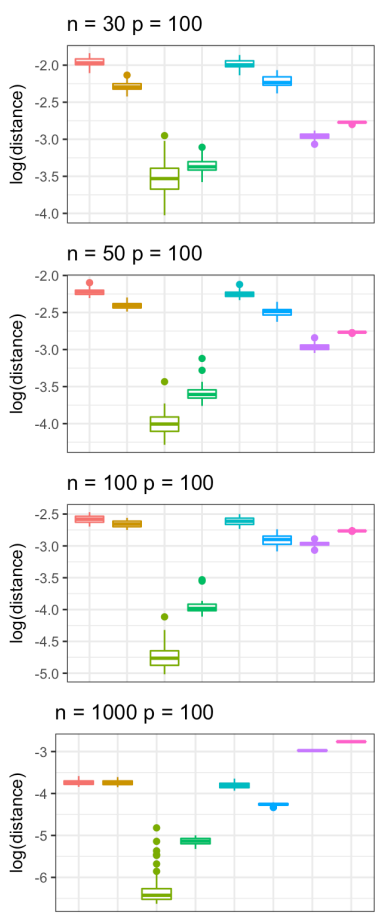

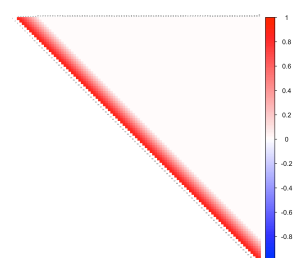

Toeplitz correlation

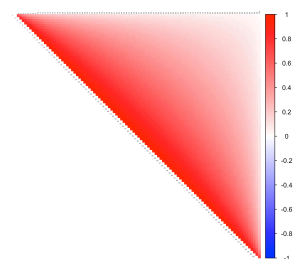

Banded precision
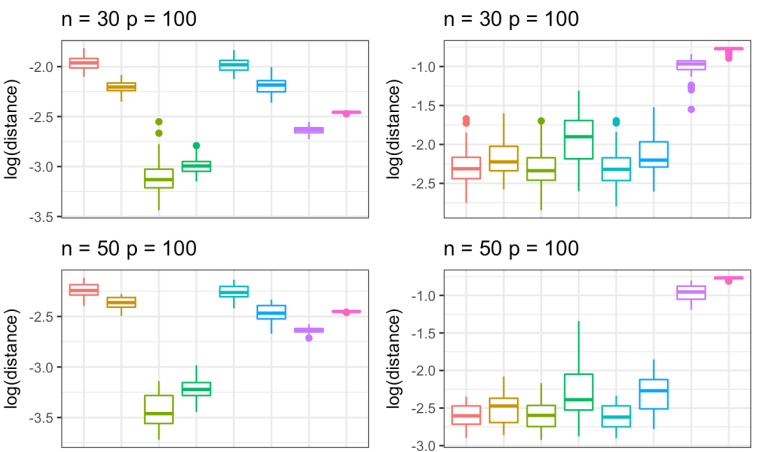

$\mathrm{n}=100 \mathrm{p}=100$
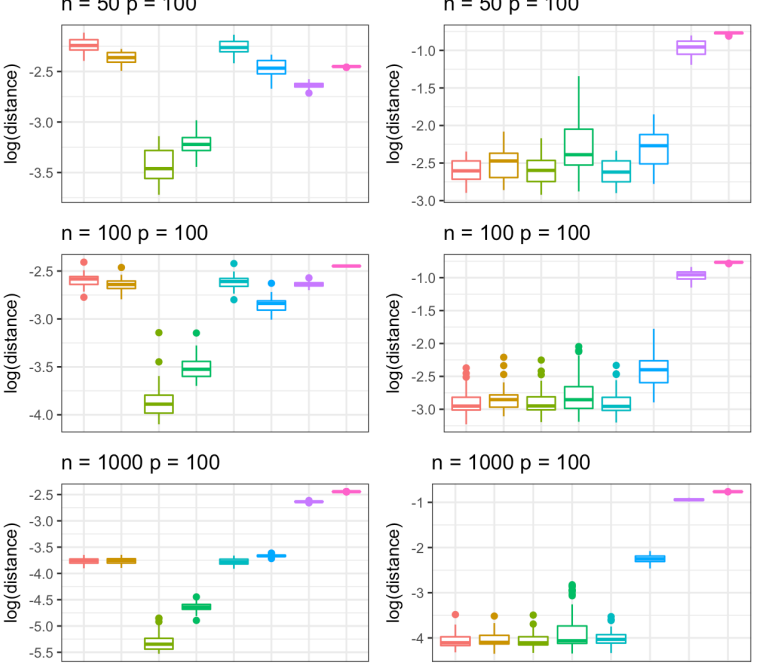

$\mathrm{n}=100 \mathrm{p}=100$
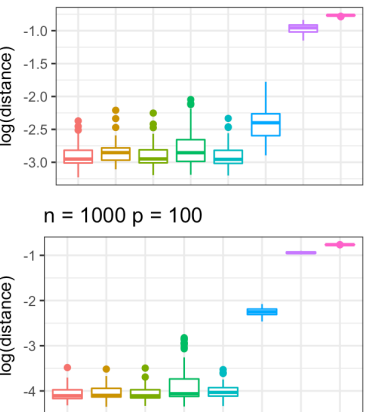

method

官 empirical

宁 corpcor

宁 corshrink

它 pdsce

由 glasso:1e-02

官 glasso:0.1

由 glasso:0.5

蒋 glasso:1

Figure S3. Box plot of the Frobenius distance between population correlation matrix and the estimated matrix using different methods - corpcor Schäfer and Strimmer, 2004 2005, CorShrink, PDSCE Rothman, 2012, GLASSO Friedman and others, 2008 and the empirical pairwise correlation matrix - for different structural assumptions on the underlying population correlation, hub structure, Toeplitz structure and the banded precision matrix structure. Image plots for the population correlation matrices are shown in the topmost panel. GLASSO was fitted using a broad range of tuning parameters, and their relative performance was also compared. CorShrink outperforms the other methods for the structured/sparse covariance models (hub and Toeplitz), closely followed by PDSCE. 
Hub correlation

correlation

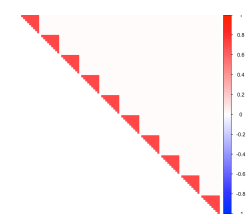

partial correlation
Toeplitz correlation
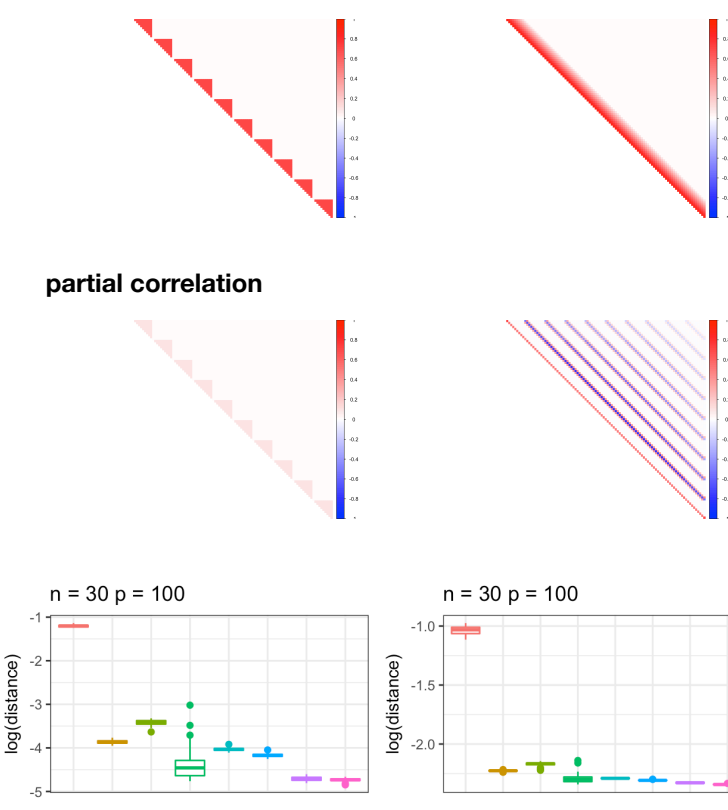

$n=50 p=100$

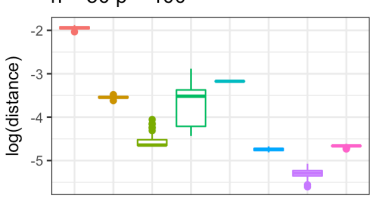

$\mathrm{n}=100 \mathrm{p}=100$

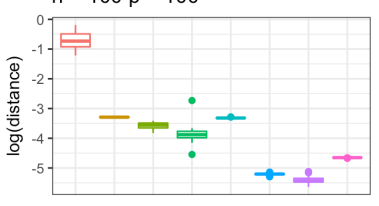

$\mathrm{n}=1000 \mathrm{p}=100$

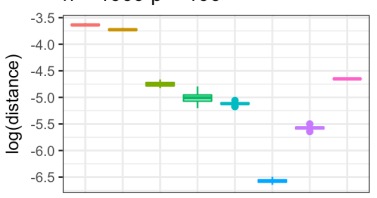

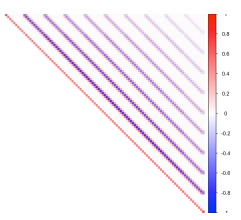
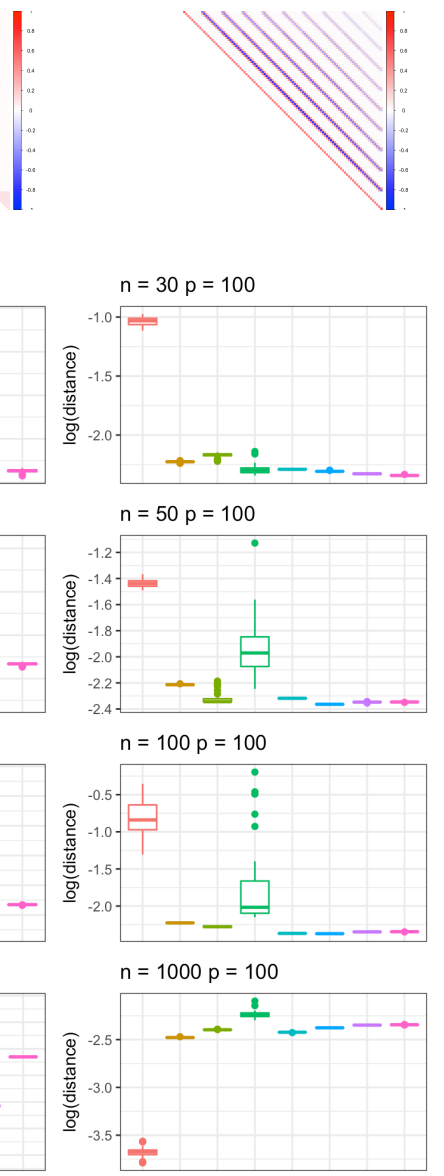

\section{Banded precision}
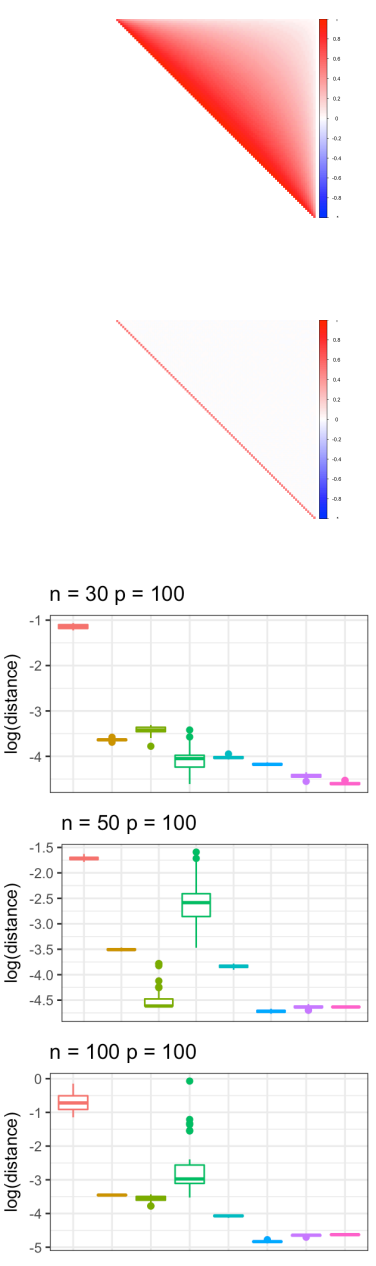

$\mathrm{n}=1000 \mathrm{p}=100$

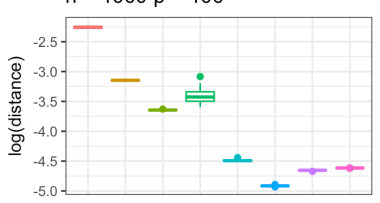

$\biguplus_{\text {empirical }}$ 官 corpcor 宁 corshrink Đpdsce 官 glasso:1e-02 官 glasso:0.1 glasso:0.5

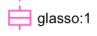

Figure S4. Comparing the accuracy (using Frobenius distance with respect to population partial correlation matrix) of the estimated partial correlation matrix from CorShrink relative to the partial correlation matrix obtained from correlation estimates of competing methods - corpcor Schäfer and Strimmer, 2004, 2005, PDSCE Rothman, 2012] and GLASSO Friedman and others, 2008 - where the population correlation matrix profiles are as in Supplementary Figure $[3$ with image plots of these correlation matrices shown in the topmost panel. GLASSO was fitted using a broad range of tuning parameters, and their relative performance was also compared. GLASSO expectedly performed better than the other competing methods, since it is designed to shrink inverse correlation matrices. 